\title{
Surface Morphology and Magnetic Anisotropy
}

\author{
R. A. Lukaszew ${ }^{1}$, Z. Zhang $^{1}$, V. Stoica ${ }^{2}$, R. Clarke $^{2}$ \\ ${ }^{1}$ Physics and Astronomy, University of Toledo, Toledo, USA \\ ${ }^{2}$ Dept. of Physics, University of Michigan, Ann Arbor, MI, USA
}

\begin{abstract}
Understanding the correlation between film structure and its ferromagnetic properties is very important for applications. Despite significant lattice mismatch epitaxial (001) $f c c \mathrm{Ni}$ films can be grown on $\mathrm{MgO}$ substrates using sputtering or molecular beam epitaxy (MBE). For both types of films it is observed that the average magnetization switching field is very similar but its azimuthal dependence is not. Structural characterization indicates very similar structure for both types of films where subtle differences are responsible for the striking difference in the anisotropy of the magnetic properties.
\end{abstract}

\section{STUDIES ON EPITAXIAL (001) NI FILMS}

We have shown that epitaxial single-crystal magnetic thin films may be used in magnetic devices such as spin-dependent tunneling applications [1]. In general, the physical properties, particularly the anisotropy [2], of epitaxial thin films are dominated by the crystallographic structure of the metal/substrate interface as well as the surface quality. In addition, for many technological applications, the roughness at the surface must be very small. To that end, we have considered the growth of $\mathrm{Ni}$ films on $\mathrm{MgO}$ substrates, which can be prepared with very smooth surfaces [3]. Theoretical studies have indicated that for $\mathrm{Ni}$ films grown on $\mathrm{MgO}$ substrates, $\mathrm{Ni}$ is expected to strongly interact with $\mathrm{MgO}$ [4]. Various researchers have studied the orientation of $\mathrm{Ni}$ films on $\mathrm{MgO}$ substrates under various growth conditions [5], and some reports indicate that $\mathrm{Ni}$ may form an epitaxial relationship with $\mathrm{Ni}[001] / / \mathrm{MgO}[001]$ and $\mathrm{Ni}(010) / / \mathrm{MgO}(010)$ for films deposited using de sputtering on $\mathrm{MgO}$ substrates held at $100^{\circ} \mathrm{C}[6]$.

In the following, we present our studies on the molecular beam epitaxy (MBE) growth/annealing and surface, structural and magnetic characterization of single domain $\mathrm{Ni}$ films grown on (001) oriented $\mathrm{MgO}$ substrates. We compare the films with similar ones grown using DC sputtering.

Ni films were grown in an MBE VG $80 \mathrm{M}$ system with a background pressure $<5 \mathrm{x}$ $10^{-11}$ torr. Ni was evaporated from a $99.999 \%$ pure source. The deposition rate was $0.5 \AA / \mathrm{sec}$. The substrates used in the experiment were $0.5 \mathrm{~mm}$ thick, $1 \times 1 \mathrm{~cm}^{2}$ prepolished $\mathrm{MgO}$ (001) oriented single crystals, which were heat-treated in UHV at $800^{\circ} \mathrm{C}$ for $1 \mathrm{hr}$. The combination of flat polished substrates and the UHV heating cycle to allow the surface layers to regain crystalline order has been proven to permit growth of single crystal metal films as well as exhibiting sharp reflection high-energy electron diffraction (RHEED) from the $\mathrm{MgO}$ surface. 
Ex-situ atomic force microscopy (AFM) characterization of the annealed surfaces indicated smooth surfaces with a root mean square (rms) surface roughness of $0.2 \mathrm{~nm}$ for (001) oriented $\mathrm{MgO}$ substrates. The substrate temperature was then lowered to the appropriate deposition temperature for metal growth (i.e. $150^{\circ} \mathrm{C}$ ). Heat transfer was by direct radiation between the heater and $\mathrm{MgO}$ substrate.

Similar growth and annealing conditions were used to grow epitaxial films using DC sputtering in a different UHV chamber. For the present study the thickness of both types of films was in the $25-50 \mathrm{~nm}$ range.

RHEED patterns were recorded continuously during MBE deposition and during subsequent annealing of the films. The RHEED pattern of heat-treated (001) $\mathrm{MgO}$ substrates showed long streaks characteristic of a smooth, single-domain surface. Sharp Kikuchi lines indicated long-range lateral coherence. The Ni RHEED pattern evolved from wide and diffuse streaks at the beginning of the growth into sharper and spotty streaks indicating three-dimensional growth [Fig. 1(a)]. The RHEED pattern for a $30 \mathrm{~nm}$ thick film indicated single crystal structure for all azimuthal orientations.

The surface morphology of the as-deposited and annealed films was also determined in-situ with scanning tunneling microscopy. The mounded quality of the surface was corroborated with in-situ STM. The rms surface roughness of the asgrown films was $0.5 \mathrm{~nm}$. In order to further smoothen the surface, the films were annealed in UHV at $573 \mathrm{~K}(\sim 1 / 3$ of the Ni melting temperature) for several hours.

Sharpening of the RHEED pattern during annealing indicated a better crystalline quality as well as smoothening of the surface. It also showed the presence of halforder streaks [Fig. 1(b)] typical of a (2x1) surface reconstruction.
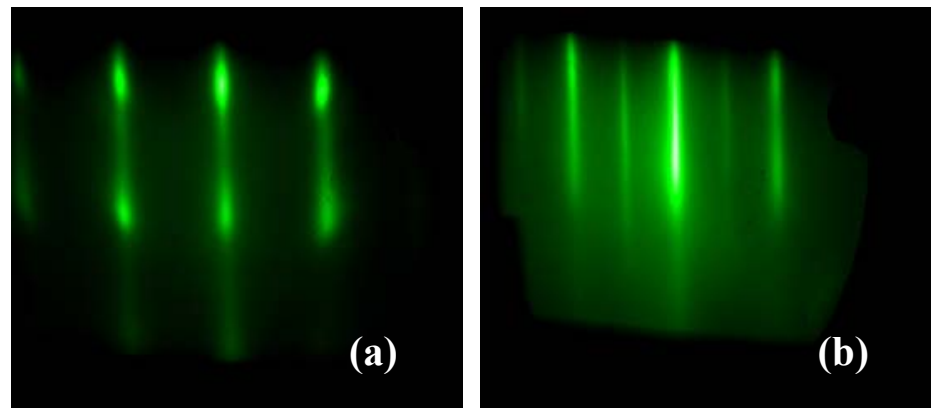

FIGURE 1. (a) RHEED pattern of the "as grown" (001) Ni film. (b) RHEED pattern of the same film after annealing. Notice the $2 \times 1$ reconstruction

Previous studies have indicated that annealing the films at a temperature approximately equal to $1 / 3$ of the melting point $(\mathrm{K})$ favors surface diffusion mechanisms leading to smoother surface [7]. STM imaging of the annealed surface shows that in this case the annealing process was dominated by "turbulent step flow" due to the presence of defects, mainly screw dislocations [Fig. 2 ]. The rms surface roughness of the annealed films was $0.2 \mathrm{~nm}$. 

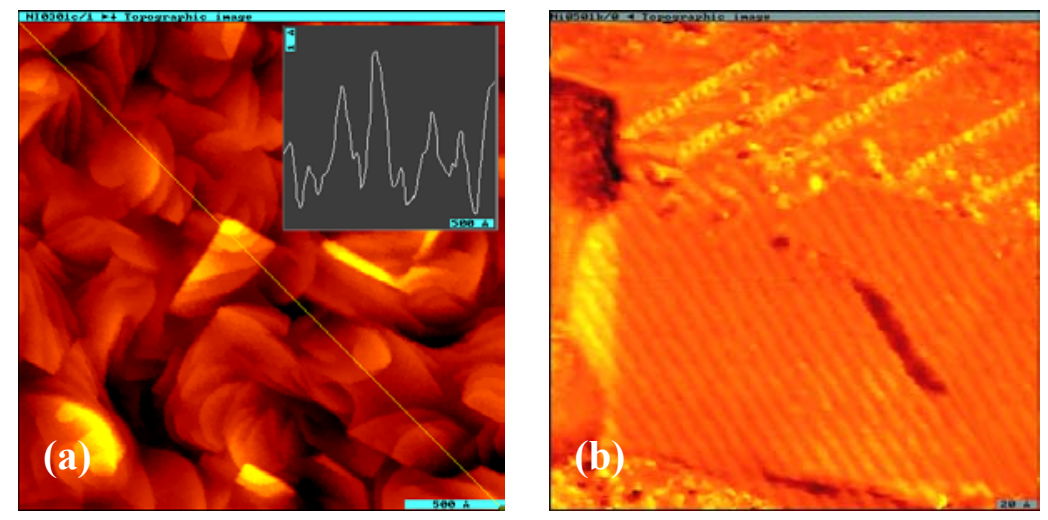

FIGURE 2. (a) STM image of the (001) Ni surface after annealing in UHV at $573 \mathrm{~K}$. The scale bar corresponds to $50 \mathrm{~nm}$. Inset: line-scan along the diagonal of the STM image. Scale-bar: $50 \mathrm{~nm}$. (b) same surface imaged with higher magnification. Scale-bar: $2 \mathrm{~nm}$.

Higher magnification of the annealed surface showed periodic nano-patterning [Fig 2 (b)] with two periodicities. A longer period $(\sim 2.1 \mathrm{~nm})$ and a shorter one $(0.7 \mathrm{~nm})$, the latter consistent with the $(2 \times 1)$ reconstruction observed with RHEED.

The $(2 \times 1)$ missing row reconstruction has been observed before, in Mo films grown on $\mathrm{MgO}$, and ascribed to the presence of oxygen at the surface [8]. Assuming oxygen diffusion from the substrate, the oxygen atoms may be positioned in three-fold coordinated sites on the Ni $f c c$ lattice, which are shifted off the top position on the top Ni layer towards the underlying Ni layer. Thus, the top Ni atoms are surrounded by oxygen, which prevents the formation of a complete top Ni layer, hence the missingrow type surface reconstruction.

As mentioned above, similar films were grown using sputtering. Structural comparison between the two types of films was done ex-situ using high-resolution XRay Diffraction, which indicated that both films had epitaxial (001) orientation with respect to the substrate, with no indication of texture or strain (Figure 3). Thus the epitaxial growth was dominated by the crystal field of the substrate.
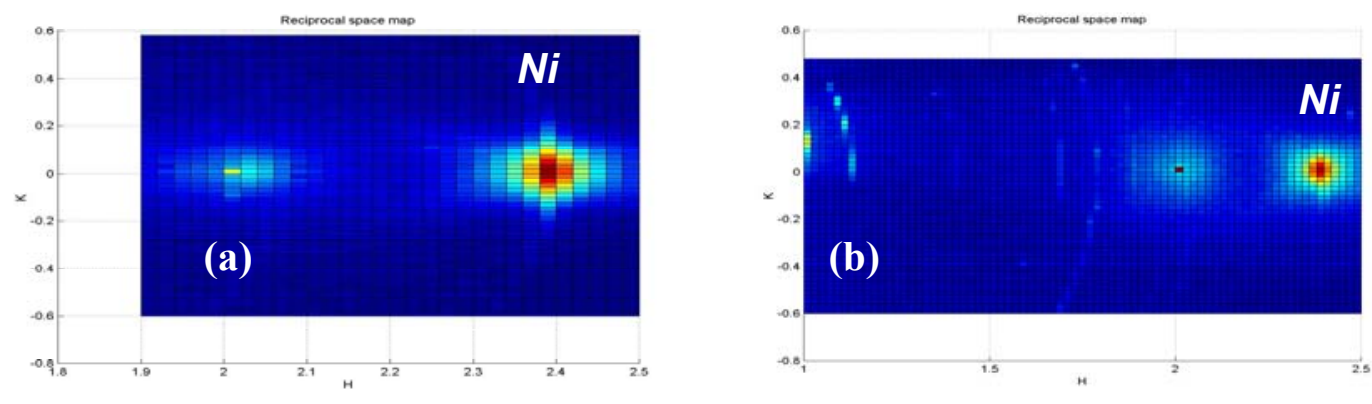

Figure 3. Reciprocal space maps obtained with High-resolution X-ray diffraction at the Advanced Photon Source (APS) showing epitaxial cube on cube growth for: (a) 30nm Ni film MBE grown; (b) $30 \mathrm{~nm} \mathrm{Ni}$ films sputtered on (001) MgO. 


\section{Magnetic Characterization}

The magnetic characterization of the films was done using ex-situ longitudinal Magneto-optical Kerr Effect (MOKE). In particular we studied the azimuthal dependence of the magnetization reversal in order to probe the different anisotropies present. Figure 4 shows the azimuthal dependence of the coercive field for both types of samples.

(a)

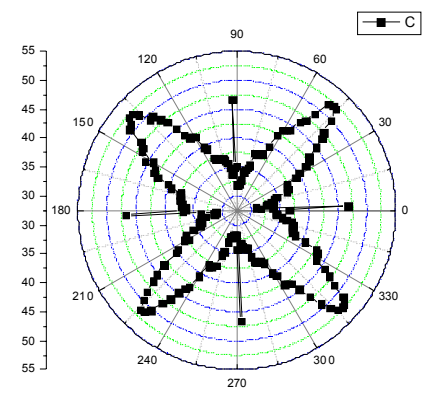

(b)

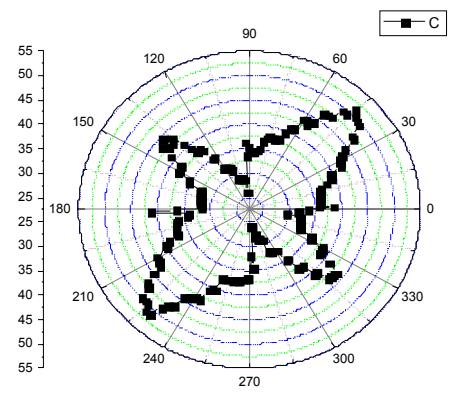

Figure 4. Polar plot of coercive field determined with longitudinal MOKE for: (a) 30nm Ni films sputtered on $\mathrm{MgO}$. Note that only 4-fold symmetry is evident. (b) 30nm Ni film MBE grown on MgO. Note the superimposed strong uniaxial anisotropy on the expected 4-fold symmetry due to the magnetocrystalline anisotropy in (001) fcc symmetry.

The magnetic anisotropy properties yield in this case significant hints regarding subtle differences in the structure of both types of films. We notice a strong uniaxial anisotropy superimposed to the expected four-fold symmetry due to magnetocrystalline anisotropy for MBE grown (001) oriented $f c c$ films. This uniaxial anisotropy is absent in sputtered films.

To explain this additional uniaxial anisotropy we postulate the presence of a highly ordered $\mathrm{NiO}$ interfacial layer in the case of MBE grown films. We speculate that this layer may not be so highly ordered in the case of sputtered films due to the higher energy of the incoming adatoms precluding the possibility of highly ordered deposition. Further, as the magnetic characterization was performed ex-situ, it is unlikely that the observed difference in the magnetic anisotropy is due to surface oxidation of the films.

This assumption is justified by the fact that $\mathrm{NiO}$ has a cubic $f c c$ crystal structure above its Néel temperature $T_{\mathrm{N}}\left(T_{N}=240^{\circ} \mathrm{C}\right)$. Below the Néel temperature there is a slight distortion of the $\mathrm{NiO}$ lattice in a (111) direction $\left(\Delta \mathrm{l} / 1=4.5 \times 10^{-3}\right)$ [9]. A strong negative uniaxial anisotropy accompanies the contraction, resulting in an easy plane defined by $\mathrm{K}_{1}$ approximately $10^{6} \mathrm{erg} / \mathrm{cm}^{3}[10]$.

Thus the uniaxial anisotropy observed in Fig. 4(b) may be associated with structural deformation of the ordered $\mathrm{NiO}$ layer upon annealing at $300^{\circ} \mathrm{C}$. Additional tests to corroborate this assumption are currently under way.

We also postulate that oxide formation has also occurred at the surface via oxygen diffusion from the interface as evidenced by the $2 \times 1$ reconstruction in the STM image 
as well as in RHEED. The lattice parameter of $\mathrm{NiO}$ is very similar to that of $\mathrm{MgO}$ and therefore was not observed in the XRD data.

\section{Conclusion}

STM characterization of annealed epitaxial (001) Ni films indicated "missing row" (2x1) reconstruction, also observed with RHEED. This reconstruction can be understood assuming the presence of oxygen segregated from the substrate to the surface during annealing. Further evidence of ordered $\mathrm{NiO}$ at the interface is obtained from the magnetic characterization of the samples, where we observed superposition of uniaxial anisotropy to the expected 4-fold anisotropy. We did not find evidence of highly ordered $\mathrm{NiO}$ in the sputtered films. Further studies to corroborate our interpretation are currently under way.

\section{ACKNOWLEDGMENTS}

The authors whish to thank Don Walko and Codrin Cionca from the MHATTCAT7 at the Advanced Photon Source, Argonne National Lab., for their assistance in

the obtention of the HR-XRD data as well as the support of the University of Toledo, Toledo, $\mathrm{OH}$.

\section{REFERENCES}

1. R. A. Lukaszew, Y. Sheng, C. Uher, and R. Clarke, Appl. Phys. Lett. 75, 1941 (1999).

2. K. B. Hathaway and G. Prinz, Phys. Rev. Let. 47, 1761 (1981); R. A. Lukaszew, E. Smith, R. Clarke, submitted to Phys. Rev. B.(2003)

3. S. S. Perry, P. B. Merrill, Surf. Sci. 383, 268 (1997).

4. G. Pacchioni, N. Rösch, J. Chem. Phy. 104, 7329 (1996).

5. H. Qiu, H. Nakai, M. Hashimoto, G. Safran, M. Adamik, P.B. Barna, E. Yagi, J. Vac. Sci. Technol. A 12, 2855 (1994); J. P. McCaffrey, E. B. Svedberg, J. R. Phillips, L. D. Madsen, J. Cryst. Growth 200, 498 (1999).

6. E. B. Svedberg, P. Sandström, J. -E. Sundgren, J. E. Greene, L. D. Madsen, Surf. Sci. 429, 206 (1999).

7. R. A. Lukaszew, Y. Sheng, C. Uher and R. Clarke, Appl. Phys. Letters 76, 724 (2000).

8. $\quad$ A. Zur, T. C. McGill, J. Appl. Phys. 55, 278 (1984).

9. G. A. Slack, J. Appl. Phys. 31, 1571 (1960).

10. J. Sievers and M. Tinkham, Phys. Rev. 129, 1566 (1962). 